\title{
REPRESENTAÇÕES DIGITAIS E INTERAÇÃO INCORPORADA: UM ESTUDO ETNOGRÁFICO DE PRÁTICAS CIENTÍFICAS DE MODELAGEM COMPUTACIONAL
}

Marko Monteiro

\section{Introdução}

As interpretações usuais a respeito das visualizações científicas, especialmente entre os próprios cientistas que as utilizam, são de que a tradução para imagens de conjuntos muito grandes de dados possibilitaria uma apreensão mais direta das complexas relações entre as variáveis em jogo, algo que seria muito difícil ou mesmo impossível de compreender de outra forma. O próprio campo acadêmico da visualização científica, como ficou claro no decorrer da etnografia que dá origem a este artigo, é voltado para a tradução de imensas quantidades de dados numéricos para imagens, a partir da premissa de que os dados podem assim ser interpretados pelos cientistas de uma forma mais direta. Desde os primeiros modelos em 3D de moléculas em madeira ou plástico, ou mesmo os moldes em cera (moulages) usados no século XIX para representar estados patológicos nos estudos de anatomia (Schnalke 2004), até os modernos modelos de partículas subatômicas ou dos padrões de gases na galáxia, representações visuais têm sido fundamentais na produção do conhecimento em diversas especialidades.

O crescimento recente do emprego das visualizações digitais (Francoeur \& Segal 2004; Myers 2008), geradas com o auxílio dos chamados "supercomputadores", em projetos científicos de grande porte, tem levado cientistas a especularem sobre como usar tais recursos para responderem a questões crescentemente ambiciosas, incluindo a previsão de padrões de clima e o funcionamento detalhado dos processos biológicos do corpo (Bement Jr. 2007). Mais do que uma forma de produção de conhecimento, modelos e representações geradas por computadores são ferramentas poderosas de intervenção na natureza e na biologia humana. Isto porque legitimam interpretações do real e, como no projeto aqui analisado, compõem ferramentas de intervenção na própria materialidade dos corpos e objetos que tornam visíveis. Por esta 
razão, as abordagens analíticas adequadas para compreender tais imagens (incluindo teorias da antropologia, sociologia, análise do discurso, comunicação, estudos sociais de ciência e estudos visuais em geral) devem dar conta não somente da construção de sentidos por meio da imagem, mas também das formas pelas quais os cientistas interagem com tais imagens, e como essas interações participam da produção do conhecimento. Além disso, tais análises devem levar em consideração as práticas de intervenção que obtêm sua legitimidade a partir do conhecimento gerado dessa forma.

Nesse contexto, investigações etnográficas de práticas de produção de visualizações científicas podem ajudar na compreensão do complexo trabalho comunicativo e representacional necessário para produzir e interpretar essas imagens, além da sua relação com a realidade que descrevem e buscam tornar visível. As investigações de práticas de laboratório têm demonstrado a importância central de inscrições científicas (Latour \& Woolgar 1986) enquanto "móveis imutáveis" (Latour 1990) que circulam do empírico para as páginas de periódicos acadêmicos, ajudando assim a desenvolver verdades científicas através de um trabalho representacional (Latour 1995). Ainda que os estudos de representações visuais na ciência tenham crescido em quantidade, diversidade e importância (Lynch \& Woolgar 1990; Lynch 2006), muitas áreas de estudo permanecem inexploradas, como as imagens digitais e os modelos computacionais em 3D. Tais investigações são relevantes atualmente, num contexto de aumento de importância de modelos computacionais e do uso de técnicas de visualização científica auxiliada por computadores nas práticas científicas contemporâneas.

Representações visuais de grandes conjuntos de dados tornam-se mais relevantes atualmente não somente em termos de apresentarem verdades sobre a natureza, mas também em práticas de intervenção que dependem de previsões geradas por computador. ${ }^{1}$ Muitos dos cientistas que fazem uso rotineiro de tais técnicas visuais pensam as imagens digitais como uma encarnação contemporânea da objetividade mecânica, antes representada por fotografias (Daston \& Galison 1990), na medida em que são geradas de uma forma automatizada. No entanto, etnografias da prática científica (Goodwin 1994; Ochs, Jacoby et alli 1994; Ochs, Jacoby et alli 1996; Myers 2008), estudos de cognição (Alač \& Hutchins 2004; Becvar, Hollan et alli 2005), estudos sociais da ciência (Lynch 2006) e estudos históricos (Pasveer 2006) têm demonstrado a importância do trabalho interpretativo que é necessário para a formação de sentido sobre os dados visualizados. O presente artigo insere-se nessa discussão, acrescentando uma análise de interações de cientistas com objetos visuais em 3D, e mostra a importância da interatividade e do gestual como fundamentais nas práticas interpretativas desse grupo. 
Uma importante inovação advinda de análises recentes da ciência, em conjunção com a discussão antropológica em geral, foi a de reinscrever a relevância da percepção incorporada (Csordas 1990) e das interações com os objetos digitais como parte do processo de produção e apreensão de sentido. Autores vêm demonstrando, por exemplo, como cientistas fundem seus próprios corpos com os processos que buscam explicar para seus pares através de interações corporais com as visualizações (Ochs, Jacoby et alli 1994; Ochs, Jacoby et alli 1996), revelando as interações entre cientistas e as inscrições por eles produzidas como processos cognitivos importantes por si mesmos (Alač \& Hutchins 2004). Mais especificamente, estudos de cientistas que pesquisam modelos de proteínas mostram o aspecto crucial do gesto na apreensão das formas e, por conseguinte, das funções das moléculas estudadas (Becvar, Hollan et alli 2005; Myers 2007; Myers 2008). Tais estudos atestam a importância epistemológica da cognição incorporada e de processos comunicativos nos processos de produção de conhecimento científico sobre proteínas. Em todos esses estudos, o aspecto interativo da transmissão e da apreensão de sentidos é chave para a análise de visualizações científicas, ampliando o escopo desses estudos para além do seu aspecto meramente visual.

A possibilidade de se "ver" um evento significativo através de visualizações científicas não é dado a priori, mas é a consumação de um processo que envolve uma gama de práticas discursivas historicamente constituídas (Goodwin 1994). O objetivo deste trabalho é, assim, o de analisar como os cientistas alcançam alguma medida de entendimento compartilhado (pois se trata de uma equipe multidisciplinar) no seu processo de construção de um modelo computacional de transferência de calor na próstata. A equipe analisada aqui realiza esse trabalho através de uma variedade de ferramentas representacionais (slides, imagens de ressonância magnética, gráficos gerados por computador, fórmulas matemáticas e desenhos no quadro-negro), além das suas próprias interações, entre si e entre eles e essas representações (incluindo falas e gestos).

O trabalho representacional que gera significado a partir dos objetos digitais com que trabalham os cientistas nunca é exclusivamente visual, mas é muitas vezes incorporado. Gestos são um aspecto central do trabalho científico observado etnograficamente, o que tem sido apontado em outras análises de trabalho científico com objetos digitais (Corrie \& Storey 2007). O fato de muitas dessas visualizações serem artefatos digitais em 3D gerados a partir de dados coletados experimentalmente torna necessária essa gama de interações com os objetos para complementar as explicações orais. Tais interações incluem: 
a) gestos: cientistas fazem uso do seu próprio corpo como apoio representacional, interagindo com as imagens através de gestos, como apontar, movimentar as mãos para sugerir ênfase, formas e orientação espacial, quando tais dados não estão claramente marcados nas imagens;

b) interações mediadas pelo computador: cientistas interagem com os artefatos digitais durante as suas reuniões através da mediação do hardware do computador, incluindo apontar com o mouse, arrastar imagens e janelas no espaço da tela, mudar atributos das imagens e dos objetos virtuais (opacidade, tamanho, textura) e o campo de visibilidade.

A natureza interdisciplinar do trabalho observado nessa etnografia torna o trabalho de construção de sentidos compartilhados sobre os dados mais complexo. Objetos visuais ajudam a estabelecer um campo de entendimento comum (Kraut, Fussell et alli 2003), mas poucos estudos analisam em detalhe as formas pelas quais esse processo ocorre, ou as suas dificuldades e limitações. Através de uma etnografia detalhada de um grupo de cientistas, para o qual o uso de ferramentas de visualização é central para o trabalho científico realizado, este artigo busca acrescentar o entendimento dos processos cognitivos do entendimento científico que ali ocorrem, que possuem papel fundamental em colaborações científicas interdisciplinares.

\section{Práticas de representação científicas}

Estudos de representação na ciência datam dos anos 1980 e 1990, quando da emergência dos Estudos Sociais da Ciência e da Tecnologia (ESCT) em geral (Daston \& Galison 1990; Knorr-Cetina \& Amann 1990; Latour 1990; Lynch \& Woolgar 1990). As análises acerca dos aspectos visuais das práticas científicas, que tornam objetos e fenômenos naturais "visíveis" ao mesmo tempo em que os tornam "conhecidos", são assim um tema tradicional desta disciplina (Latour 1995; Lynch 2006; Pauwels 2006).

Pouco discutidos nessa literatura são os modelos em 3D como ferramentas centrais da ciência contemporânea, cada vez mais dependente de uma infraestrutura computacional potente (Chadarevian \& Hopwood 2004), as formas pelas quais o conhecimento é construído na interação com tais objetos (Myers 2007) e o campo emergente dos modelos computacionais em $4 \mathrm{D}$, que incorporam a dimensão temporal. A busca para construir modelos capazes de prever o desenrolar de fenômenos da natureza contextualiza a forma pela qual são utilizados como ferramentas de classificação, interfe- 
rência e manipulação dos objetos naturais que procuram descrever, ou seja, a ideia de que novas ferramentas tecnológicas podem, ao incorporarem modelos computacionais, prever o futuro de uma cirurgia, altera o caráter dos novos tratamentos desenvolvidos, legitimando assim formas de intervenção automatizadas.

Visualizações científicas são centrais para as práticas científicas de "revelação" das propriedades internas de objetos da natureza, tornando-os assim analisáveis por modelos teóricos (Lynch 1990), isto é, o processo de construção de um saber científico sobre um objeto passa, entre outros elementos, pela construção de visualizações, que são assim esquematizações visuais do conhecimento produzido a respeito de um objeto. Tais imagens não são, portanto, somente visualizações: elas buscam estabilizar uma definição conceitualmente válida da ordem interna de determinado objeto ou fenômeno da natureza.

Em outras palavras, elas constroem uma descrição daquelas que são consideradas, de acordo com o aparato conceitual em jogo, as suas qualidades essenciais (Lynch \& Woolgar 1990). Tornar um objeto visível através de visualizações científicas é também, neste contexto, torná-lo mensurável (Latour 1990; Lynch 1990; Pauwels 2006) através de meios representacionais que, para manterem sua "objetividade", devem manter-se estáveis, reprodutíveis e manipuláveis na forma dos "móveis imutáveis" definidos por Bruno Latour (1990).

Ainda que a objetividade esteja sempre em questão nas representações científicas, a fidedignidade de um modelo computacional está diretamente relacionada à sua expressão de uma "objetividade mecânica" (Daston \& Galison 1990). Tal conceito refere-se ao processo histórico pelo qual imagens produzidas mecanicamente, a partir do século XIX (fotografias, filmes), foram percebidas como as formas visuais que garantiriam a maior objetividade possível, ao supostamente retirarem do processo de produção da imagem a interferência subjetiva do ser humano.

Modelos tridimensionais são tipos de representação científica particulares pela forma holística que mostram um objeto, tornando possível a visualização de relações internas a ele (Lynch 1990), acrescentando a isto a possibilidade de uma percepção tátil e espacial do objeto (Hopwood \& Chadarevian 2004). A especificidade de tais modelos enquanto ferramentas de representação advém, igualmente, da sua habilidade de simular a natureza: o cientista deve, através do modelo, ser capaz de visualizar um mundo possível (Pauwels 2006), tendo em vista uma correta reprodução de propriedades fundamentais de determinado objeto, que podem ser representadas no decorrer do tempo. A percepção tátil e espacial dos modelos é também 
um fator na manipulação de objetos virtuais em 3D, como demonstram os exemplos abaixo.

Práticas de representação na ciência podem ser analisadas a partir de um quadro analítico da "ação como cognição" (Alač \& Hutchins 2004), que descreve a forma pela qual os cientistas dependem de interações incorporadas às representações por eles produzidas a fim de comunicarem seus resultados aos pares e darem sentido a realidades físicas e biológicas de maneira colaborativa. Essas interações estabelecem assim uma correspondência entre as realidades e os modelos. O aspecto incorporado da relação entre os cientistas e os artefatos digitais como uma forma de cognição e de comunicação de sentido é uma área pouco estudada no campo de análise dos ESCT. A ideia de que a fala dos cientistas interage com visualizações e gestos para constituir o significado enquanto prática cognitiva (Ochs, Jacoby et alli 1994; Ochs, Jacoby et alli 1996) é, no entanto, crucial para compreender como a gama de atividades visuais e gestuais que ocorrem nas reuniões aqui discutidas constrói a visibilidade dos objetos estudados. As interações incorporadas são, desta maneira, um aspecto central da própria construção do conhecimento de forma interdisciplinar e colaborativa.

Além disso, tais estudos ajudam a revelar como práticas científicas envolvendo objetos virtuais possuem características conceituais e perceptivas (Becvar, Hollan et alli 2005). São conceituais, pois ideias e teorias são construídas e testadas através dessas práticas; são perceptivas, pois os cientistas dependem de uma atenção para a forma e o espaço no processo de interpretação dos dados apresentados enquanto visualizações em 3D; além disso, dependem também de interações com essas visualizações para produzir sentido e compartilhá-lo. Tais sentidos assim constituídos podem tornar-se conhecimento científico, que é considerado, a partir de então, puramente teórico ou conceitual.

\section{O trabalho etnográfico}

A equipe de cientistas estudada neste trabalho está localizada numa das principais universidades públicas do sudoeste dos Estados Unidos. O grupo trabalha com dados colhidos num hospital de pesquisa localizado em outra cidade, a $258 \mathrm{~km}$ de distância, processando os dados com o auxílio de supercomputadores pertencentes à própria universidade. O grupo é formado por professores, pesquisadores pós-doutorandos e alunos de pós-graduação. Suas áreas de especialização incluem ciência da computação, engenharias civil e biomédica, matemática aplicada, mecânica computacional, visuali- 
zação científica e medicina. Os cientistas possuem diversas nacionalidades, incluindo indianos, chineses, iranianos, tchecos, poloneses, franceses e norteamericanos. A maioria deles possui uma trajetória acadêmica interdisciplinar em maior ou menor medida, com carreiras de pesquisa que abrangem uma variedade de disciplinas e interesses.

O objetivo científico do grupo é produzir um sistema computacional que possibilite previsões corretas do dano causado a tecidos vivos por calor, disponibilizando esses dados em tempo real para médicos que realizam intervenções de retirada de tumores na próstata. Essa tecnologia possibilitaria, na visão dos cientistas, um novo paradigma para terapias térmicas minimamente invasivas, utilizando-se abrasão com laser. O novo tratamento é pensado assim como uma maneira de reduzir custos, tempo de cirurgia e trauma no paciente. O intuito maior é usar imagens de ressonância magnética térmica para fornecer ao cirurgião um controle maior de intervenções com laser por retroalimentação de dados entre sua clínica e os supercomputadores. Sintomas presentes nas células (morte ou apoptose por calor) seriam utilizados para calcular em tempo real os efeitos futuros de uma cirurgia, a fim de dar ao médico a possibilidade de adequar o procedimento a cada paciente, em tese, aumentando a eficiência e reduzindo os efeitos colaterais.

A pesquisa etnográfica foi conduzida entre novembro de 2006 e março de 2008. Ela incluiu observação participante nas reuniões de trabalho semanais dos cientistas, entrevistas com todos os participantes do grupo e observações das instalações utilizadas por eles. Trinta e duas reuniões foram observadas e registros em vídeo dessas reuniões também foram analisados. Todos os cientistas foram entrevistados pelo menos uma vez. Observação participante se deu em duas palestras e em uma conferência internacional das quais o grupo fez parte. Um workshop de uma semana aconteceu nas instalações de supercomputação utilizadas pelo grupo, e duas viagens foram feitas ao hospital de pesquisa.

Os participantes do grupo fazem uso de diversas práticas de visualização científica e modelagem, aí incluídos fórmulas e símbolos (compartilhados ou não), representações geométricas de tecidos e imagens de ressonância magnética. Práticas e procedimentos de visualização (entre eles, diagramas, gráficos, tabelas e imagens digitais em 3D) são utilizados em larga escala no trabalho conjunto dos cientistas. Apresentações formais e conversas nas reuniões focam e contextualizam os problemas de entendimento a respeito do que as visualizações significam, a validade das relações apresentadas e a aceitação pelos pares dos procedimentos e dos resultados de determinado cientista.

Atividades-chave incluem, por exemplo, a leitura de um "pico" ou "depressão" num gráfico, a interpretação de uma função matemática a partir da 
linha representando os resultados que dela derivam, e a correlação destes com um entendimento mais profundo de processos biológicos ocorrendo no corpo do paciente. Desta forma, o entendimento de representações visuais advindas de uma diversidade de disciplinas (por vezes bastante díspares) e uma percepção compartilhada de que tais imagens foram produzidas mediante procedimentos válidos são a parte central do trabalho cotidiano dos cientistas.

\section{Análise: para além do visual na representação científica}

Interações com representações visuais nas reuniões de trabalho do grupo acontecem através de diversas modalidades, incluindo gestos corporais (apontar para algo, movimentar a mão para demonstrar ênfase ou acrescentar significado a uma imagem), a movimentação de objetos na tela com o mouse, a alteração de atributos visuais da imagem e a mudança de posição de um objeto durante uma apresentação. O foco deste trabalho é compreender assim a natureza interativa de como visualizações participam nas práticas dos cientistas.

Deste ponto de vista, as imagens não são meramente representacionais no sentido de mostrar de forma transparente uma realidade (simbolicamente significando algo observável na natureza), mas precisam ser interpretadas a fim de adquirirem significado no decorrer de um processo, que é interativo. No caso do grupo de cientistas aqui observado, existe o desafio adicional de superar a falta de um corpus de conceitos e de premissas compartilhadas que guiem a produção e a apresentação visual de um conjunto qualquer de dados.

Esta particularidade, característica de um grupo tão interdisciplinar, coloca ainda mais importância nos artefatos digitais como organizadores de uma referência comum de significados durante as reuniões. Ela também aumenta o trabalho interpretativo necessário para as visualizações em pauta fazerem sentido durante determinada reunião. De acordo com alguns dos cientistas entrevistados, as imagens ajudam cientistas de disciplinas diversas a compreender um resultado "só olhando". Como fica claro no decorrer da etnografia, esta percepção nativa encobre um processo que é muito mais complexo e que demanda um trabalho que vai além do visual, incluindo gesto, fala, movimentos e interações com os artefatos e as visualizações digitais.

Essas práticas comunicativas não são "visíveis" a não ser através de uma análise minuciosa dos seus processos sequenciais. Ainda que possam ser separados de forma analítica a fala, os gestos e o visual como elementos 
ontologicamente distintos, tais facetas do processo comunicativo nunca ocorrem de forma separada no decorrer das reuniões. Um cientista, ao apresentar seus dados, pode estar falando e ao mesmo tempo movimentando a sua mão; logo depois, ele pode apontar para uma parte de uma imagem e fazer um desenho no quadro-negro para complementar a explicação em curso. Tudo isto é capaz de ocorrer no espaço de poucos segundos. Mostram-se a seguir alguns exemplos desses elementos (incluindo gestos e interação) na medida em que se materializam nas interações observadas etnograficamente.

A compreensão dos complexos processos representados e discutidos nas reuniões requer, assim, que todas as modalidades de discurso trabalhem em conjunto para transmitir significado. Embora uma imagem de ressonância magnética possa revelar muito sobre a localização da próstata em relação a outras estruturas do corpo de um paciente, ela não consegue transmitir todas as informações de interesse para o cientista, incluindo a localização da sonda, a intensidade do laser, as temperaturas no interior da próstata antes e depois da abrasão do laser, ou a quantidade de dano causado ao tumor.

$\mathrm{Na}$ medida em que os cientistas não compartilham necessariamente um mesmo aparato conceitual, nem participam em conjunto de todos os experimentos envolvidos no projeto, o exercício interpretativo semanal que ocorre nas reuniões é aquele em que ocorre, na prática, a construção e a materialização desses objetivos compartilhados. A interpretação de imagens e a interação com as mesmas é, como constatado pela etnografia, um aspecto importante desse processo.

\section{Gesto}

Apontar é um dos gestos mais frequentemente utilizados por todos os cientistas em suas apresentações. Mesmo naquelas instâncias em que este gesto não acrescenta nenhum sentido adicional além de ajudar a ilustrar o que está sendo dito (por exemplo, apontar para uma fórmula ou estrutura ao mencioná-la oralmente), tal gestual ajuda a estabelecer uma conexão direta entre as palavras e as partes específicas de uma visualização. Uma prática bastante comum é apontar para uma fórmula, ou partes dela, no decorrer de uma explicação, a fim de explicitar o que cada termo significa e quais as relações estabelecidas entre eles. No exemplo 1 vemos Lynn, ${ }^{2}$ uma aluna de pós-graduação em matemática computacional e aplicada, abrindo uma reunião com uma explicação sobre as suas definições: 


\section{Exemplo 1: Explicando definições ${ }^{3}$}

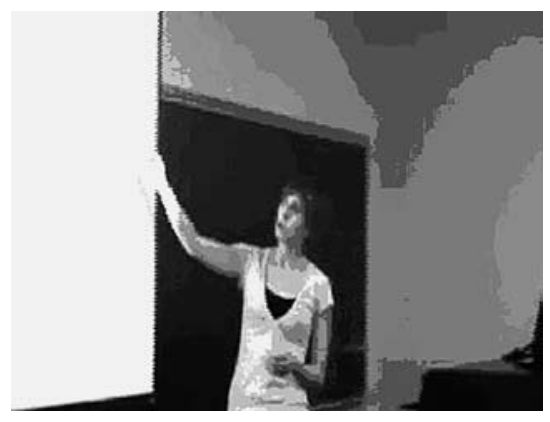

Lynn: o ponto central do nosso modelo é modelar a temperatura em mudança na próstata, isso é, o modelo da transferência de calor, e (.) nós entendemos que a temperatura vem como uma mudança de energia (.) ((desvia seu olhar do público para a tela atrás de si)) então, essencialmente, estamos aqui ((anda em direção à tela e aponta)): Q deve ser (.) ((volta seu olhar para o público)) uma mudança na temperatura será derivada de alguma forma de uma mudança de energia. $^{45}$

A premissa principal da fórmula que Lynn explica neste exemplo é a de que "mudanças em temperatura são um fator da mudança de energia" e que essa relação está corretamente descrita pela fórmula apresentada. Enquanto a fórmula está visível na tela, Lynn aproxima-se e aponta para partes específicas dela, como as variáveis ( $\mathrm{x}, \mathrm{q})$, ao mesmo tempo em que explica de que maneira serão definidos os seus valores. Seu olhar volta-se para a tela, depois para o público; as imagens na tela participam da sua explanação oral na medida em que ela dá ênfase a partes específicas das mesmas no decorrer da fala.

No exemplo 2 temos Clark, um aluno de pós-graduação em ciência da computação, explicando fórmulas visíveis na tela para o público. As fórmulas não estão claras para Mark, um professor de engenharia e mecânica computacional, que pergunta o que exatamente está sendo exposto. Clark busca esclarecer apontando para partes específicas da fórmula que mostram o que está sendo proposto matematicamente ("aquela transformação"). Lewis, o investigador principal do grupo e professor de mecânica computacional, ajuda na parte final do trecho a definir a fórmula como uma "transformação inversa de Fourier". 


\section{Exemplo 2: explicando funções matemáticas}

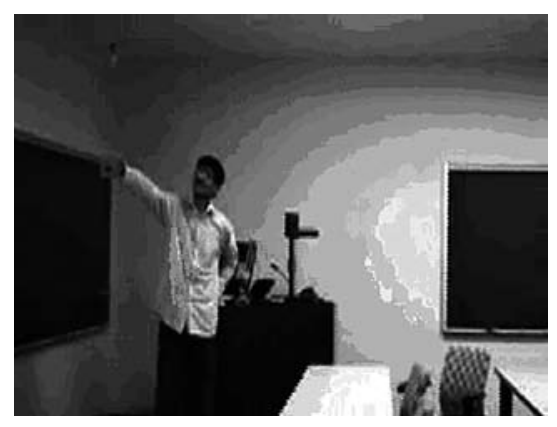

Clark: Então é esta a razão por trás ((aponta para a tela)), tipo pegar essa transformação, onde (.) isso é [na verdade]

Mark: [o que é (?)]

Clark: Hum, quero dizer ...

Mark: (?) O que é (?) a nova função $\mathrm{P}$, o que é a função antiga?

Clark: Sim, na verdade é ((olha e aponta para a tela)) a [inversa (?)]

Lewis: [Transformação inversa de Fourier]. ${ }^{6}$

Então, enquanto Clark explica a "transformação" como parte central da sua apresentação, ele aponta para a tela a fim de enfatizar ainda mais aquele aspecto. Mark tem uma dúvida ("o que é a função antiga?"), e Clark responde oralmente ("transformação inversa de Fourier"), ao mesmo tempo apontando para o lugar no qual a fórmula está localizada na própria tela. As fórmulas mostradas nos slides utilizados pelos cientistas durante as reuniões são muitas vezes longas e complexas, e trocas como esta são muito comuns. Isto ilustra o fato de que uma fórmula matemática, ainda que seja pensada pelos cientistas como a forma mais direta e livre de mediações para apresentar conhecimento, envolve também alguma medida de percepção que não depende da teoria em si (por exemplo, encontrar a localização espacial no slide de uma parte específica da fórmula).

Gestos são assim muito mais do que meros indicativos de lugar num slide ou no interior de uma fórmula. Eles ajudam a transmitir significados mais abstratos, que são essenciais nos processos de explicação e apresentação de resultados que ocorrem nas reuniões. No caso desse grupo, no qual as explicações de definições ocorrem entre pares que não possuem uma trajetória intelectual compartilhada, ter certeza de que todos têm alguma medida de conhecimento sobre as definições básicas 
relativas a um conjunto de dados ou a uma metodologia é fundamental para o sucesso do esforço coletivo. Apontar para partes diferentes das fórmulas ajuda a estabelecer para os cientistas as relações entre os termos da função matemática, auxiliando assim o processo de convencimento de que determinada representação matemática de um processo biológico é adequada ou correta.

No exemplo 3 Clark explica oralmente o processo de registro de imagens. Embora a explicação seja relativamente clara, ele ilustra sua apresentação com uma série de visualizações e gestos que complementam o significado do processo. Poder-se-ia esperar que o registro, por ser um processo de manipulação de imagens, pudesse ser mais bem explicado com o uso de visualizações. Mas como se vê a seguir, o significado da metodologia só fica mais claro para o grupo através de um processo relativamente longo de explicação que envolve imagens, gestos e interações com as visualizações. O exemplo 3, ainda que longo, é relevante, pois ajuda a contextualizar também muitos dos objetivos finais do trabalho coletivo do grupo. Em outros termos, vê-se como um cientista do grupo, no processo de explicação de um aspecto específico do projeto (registro), constrói uma compreensão dos objetivos maiores do projeto:

\section{Exemplo 3: explicação verbal do processo de registro de imagens}

Clark: Primeiramente vamos... quero dizer, gostaria de relembrar o que é registro de imagens (.) Bem, na visão computacional (.2) quando conjuntos de dados são obtidos por amostragem de uma mesma cena (.) ou, quero dizer, então, se você, se nós adquirimos os dados visuais (.2) da mesma cena, em períodos diferentes (.) ou se adquirimos os dados sobre uma imagem a partir de perspectivas diferentes, então essas duas coisas estarão em coordenadas diferentes. (.) E precisamos, o registro de imagens é o processo de registrar ambos num mesmo sistema de coordenadas. (.) E é necessário pois para comparar ou integrar esses dados... precisamos tê-los no mesmo sistema de coordenadas. Por exemplo, no nosso caso, no qual estamos fazendo ... no qual teremos imagens da próstata, como sabemos, dados visuais da próstata anteriores nos serão dados... muito antes da operação. E então, durante o dia da operação nós teremos ... dados da próstata novamente. Então (.) quero dizer para fazer sentido da ... desses dados, e para realizar cálculos nesses dados, precisamos ter realizado o registro dessa nova imagem da próstata sobre a imagem anterior, na qual nós já havíamos realizado alguns cálculos prévios. Então, é por esse motivo que precisamos realizar o registro das duas imagens da próstata. ${ }^{7}$ 
Enquanto explica oralmente o processo de registro de imagens, Clark mostra na tela um slide com exemplos visuais: uma sequência de três imagens. As duas primeiras referem-se à mesma paisagem, mas cada imagem apresenta uma parte separada com alguma sobreposição. Para determinar os pontos de sobreposição, um código de registro é necessário. A terceira imagem é o resultado do processo de registro, mostrando as duas imagens e os pontos de sobreposição em relação ao cenário que representam.

Enquanto fazia sua explanação oral, Clark estava estático, falando do pódio ou de pé ao lado dele, sem muito movimento corporal. Os slides contêm somente palavras e símbolos matemáticos. Ao chegar, na sequência da apresentação, ao slide contendo as três imagens explicadas acima, Clark caminha até a tela e começa a interagir com elas. Para ilustrar como uma imagem, a fim de ser "registrada", precisa sofrer uma rotação, Clark vai diretamente à tela e movimenta sua mão de forma análoga ao movimento descrito em sua explicação, como mostra o exemplo 4:

\section{Exemplo 4: fazendo as imagens se moverem através do gesto}
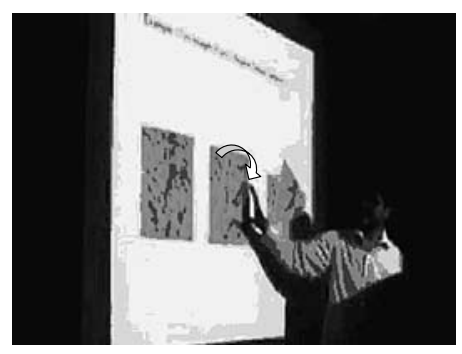

Clark: Então a ideia é tipo girar ( (faz o gesto com a mão enquanto aponta para a segunda imagem) $).^{8}$

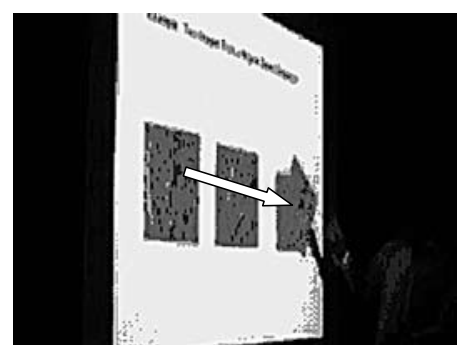

...essa imagem de forma que ela se sobreponha ( $(a$ mão desloca-se para a terceira imagem, registrada) $)^{9}$

Clark "gira" uma das imagens usando suas mãos, depois movimenta a mão pela tela para mostrar o caráter sequencial do seu exemplo visual (imagem a, imagem b, imagem registrada). A compreensão do giro sofrido pela imagem é fundamental, pois sua explicação refere-se a como as imagens, ainda que visualmente diferentes, mostram partes diversas de um mesmo cenário, e de como essa unidade pode ser recomposta matematicamente. A fim de combinar as duas imagens, um código deve computar a correta orientação da segunda imagem em relação à primeira. Assim, o público, para 
compreender o processo, precisa fazer a conexão entre as imagens geográficas que Clark usa na explanação e as imagens de ressonância magnética da próstata que serão usadas no projeto.

Seu gestual sugere o movimento necessário para conseguir a combinação desejada (rotação), além de estabelecer uma relação entre as três imagens (imagem c enquanto compósito das imagens a e b). O movimento da sua mão também demonstra que, pelo menos no exemplo em questão, as duas imagens mostram partes diferentes de uma mesma paisagem, com diferentes orientações. Ao "girar" a imagem com o seu gesto, Clark transmite, de forma interativa, mais significado do que através de uma explanação oral ou do uso de um exemplo visual estático.

\section{Interagindo com artefatos digitais através do computador}

Interações com objetos digitais são importantes para a transmissão de significados nas reuniões dos cientistas. Tais interações podem ocorrer, como mostra o exemplo 4, diretamente entre o apresentador e a imagem projetada na tela. Outra forma importante de interação com imagens e objetos virtuais é a sua manipulação através do computador. Isto inclui clicar partes da imagem, arrastar janelas (para dar ênfase, ou mudar sua posição em relação a outros elementos que estão sendo mostrados), mudar cores e outros atributos das imagens (como tamanho e opacidade), entre outros. Todas essas interações que acontecem nas reuniões têm como intenção enfatizar aspectos de interesse do apresentador, tornar afirmações mais claras (por exemplo, apontando com o cursor ou realçando determinada imagem) e complementar os significados, o que é transmitido oralmente ou pela escrita nos slides.

No exemplo 5, Clark continua sua explicação do processo de registro de imagens. Na medida em que a reunião avança, ele alterna de uma interação feita principalmente com a tela para a manipulação das imagens, de forma direta, por meio do computador. No exemplo a seguir, ele apresenta três imagens, que ele rotula de a, b, c. Cada imagem é visível como uma janela separada. A apresentação ocorre através de um programa de visualização científica que permite a manipulação direta de variáveis, tais como valores numéricos atribuídos à cor, ao tamanho, à opacidade. Vemos também Luke, outro aluno de pós-graduação em matemática computacional e aplicada, que apresenta esta parte junto com Clark, ajudando-o em termos de quais manipulações efetivar para dar curso à apresentação. 


\section{Exemplo 5: "clicando" e arrastando}
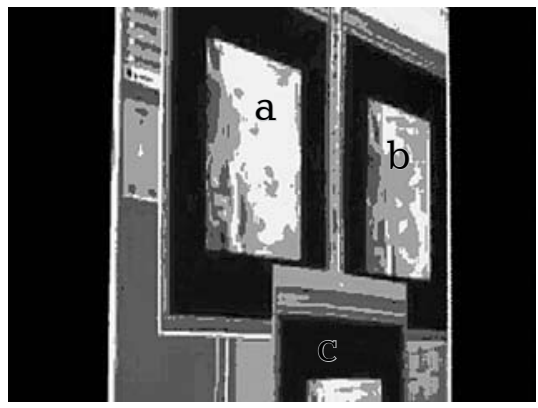

Clark: Então essas são as imagens A e B (.) e (.) então nós aplicamos a transformação em A

((enquanto está falando, Clark clica nessa janela com o mouse, trazendo-a para o primeiro plano. Ele então movimenta o mouse para usar a ferramenta do programa e conferir o valor de X))

(.13) Como dissemos X era 15.

((ele muda o valor da variável, causando uma mudança de cor)) (.14) e hum (.) $\mathrm{Y}$ era 47

((movimenta o mouse para conferir os valores de $Y))(.5)$

(...)

Então, essa comparação de imagem também fez na verdade uma sobreposição de ambas as imagens (.) ((ele se afasta do console e anda em direção à tela, apontando para a imagem na parte de baixo)). Essa aqui é a imagem transformada, e essa aqui ((aponta para a imagem no topo à direita)) é a imagem $\mathrm{B}$, e ((ele anda de volta ao console)) se nós [huum]

Luke: [Você poderia] deslocar a janela de baixo um pouco para cima?

Clark: Ok.

((ele clica e desloca a janela para cima, tornando uma parte maior da mesma visível na tela))

((durante esse tempo ele está deslocando a imagem e também movendo o cursor pela tela para mostrar as duas imagens superpostas))

$\mathrm{Ok},(?)(.8)$

((ele continua deslocando as imagens na janela de baixo, movimentando-as da esquerda para a direita, expondo como uma parte da imagem está sobreposta à outra)). ${ }^{10}$ 
No exemplo 5, fica claro como a interatividade é importante na comunicação dessas complexas operações matemáticas e visuais. A explanação oral por si só não foi o bastante para estabelecer claramente o significado desejado. Da mesma forma, as imagens em si, mostradas estaticamente (como no exemplo 4), não conseguem expressar o conteúdo em questão. As imagens estáticas demandaram um trabalho gestual para sugerir a mudança de orientação necessária ao registro das duas imagens. O gestual em conjunção com as imagens, ainda que mais completo, também não exprimiu com a completude necessária o processo de registro.

Por isso, Clark e Luke, os apresentadores dessa reunião, voltam-se para o programa de visualização a fim de mostrarem diretamente como o processo é gerido, reproduzindo as operações que fizeram nos dados brutos. O programa adotado na apresentação é o mesmo utilizado pelos alunos para efetuarem o registro nas imagens-exemplo (a paisagem) e nas imagens da próstata. Usando o programa, os apresentadores conseguiram manipular as imagens no espaço virtual enquanto explicavam as operações realizadas. Luke e Clark mostram, ao movimentar as imagens e ao mudar os seus atributos, como elas estavam sobrepostas. Isto foi feito para "confirmar visualmente", nas palavras de Luke, que os aspectos das duas imagens coincidem. Em outros termos, a validade das transformações matemáticas aplicadas às imagens pôde ser estabelecida visualmente; ao mesmo tempo, o público demonstra uma compreensão mais clara do processo após o uso dessas diversas estratégias explicativas, ajudando a convencê-los de que o trabalho realizado por Luke e Clark é cientificamente "válido".

Tais exemplos sugerem a importância dos aspectos perceptivos (Becvar, Hollan et alli 2005) no trabalho científico cotidiano desse grupo, indispensável ao lado do trabalho conceitual, mais diretamente associado à ciência enquanto tal. Paralelamente, tal qualidade perceptiva não se resume a explanações orais ou a imagens estáticas associadas às palavras na tela: a interatividade mostrada nesses exemplos é crucial no estabelecimento de relações significativas entre o que está sendo dito e o que está sendo exposto. Isto inclui interatividade entre o apresentador e a imagem na tela através de gestos, e interatividade com artefatos digitais através do computador. A "confirmação visual" final é mostrada abaixo: 


\section{Exemplo 6: visualizando o processo de registro}

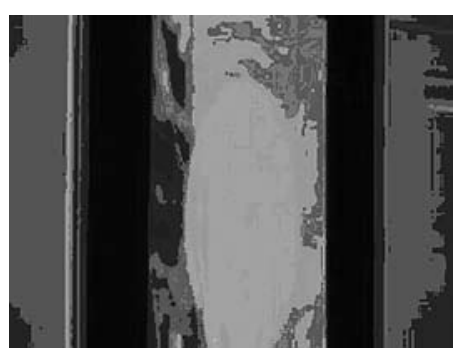

Luke: Então, quero dizer (.) o que a gente montou basicamente foi uma maneira de comparar as duas imagens para, tipo, visualmente ((enquanto está falando, ele arrasta as imagens para cima e para baixo na tela)) confirmar que o código está fazendo o que deveria estar fazendo. Então, se você olha para... ((ele manipula a imagem de baixo e, agora, no lugar de uma linha vertical separando as duas partes da imagem, vê-se uma abertura circular, que expõe a separação entre as duas imagens sobrepostas. Manipulando essa abertura, ele a torna maior ou menor, mostrando como a imagem de cima coincide com a imagem de baixo))... ${ }^{11}$

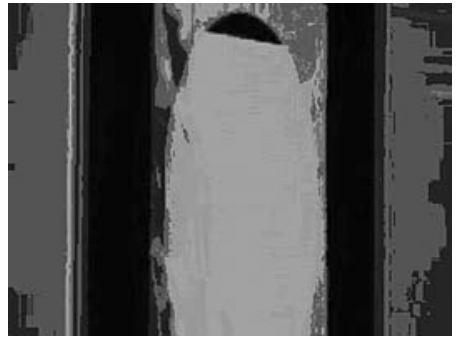

... o corte, b logo aqui, e depois, hum, o círculo mais claro no meio é a imagem registrada $\mathrm{A}, \mathrm{e}$... ( (ele abre e fecha o círculo, expondo a superposição das imagens)) dá pra ver que o código está (.) está tipo visualmente fazendo o que esperaríamos que fizesse. ${ }^{12}$

A interatividade ocorre, como mostram os dados etnográficos, no decorrer de toda a explicação do processo de registro de imagem. O movimento de abrir e fechar o círculo, que depende da manipulação de Luke através do software do computador, evidenciando como as imagens sobrepostas são "iguais", é muito mais significativo para o público do que todas as formas anteriores de explicação que foram tentadas. Esta demonstração teve mais efetividade em tornar claro o procedimento do que a explicação oral da teoria por trás do processo, ou do que apresentar a sequência de imagens estáticas das diferentes fases do processo de registro. 
Outros momentos interativos que podem ser enfatizados aqui são o movimento das janelas, a fim de enquadrá-las melhor em relação aos outros elementos da tela, enfatizando alguns e tirando a ênfase de outros; e clicar uma janela que contém uma imagem para esclarecer dinamicamente a ênfase dada a cada elemento enquanto a explanação oral está acontecendo.

\section{Interagindo com objetos digitais}

Interagir com objetos digitais pode ajudar a explicar uma metodologia (como na que foi feita do registro de imagens), mas pode também facilitar a apresentação de resultados experimentais. A interação dos cientistas com "inscrições" digitais (Latour \& Woolgar 1986; Latour 1990) produzidas a partir de experimentos ajuda assim a transmitir as relações entre diferentes aspectos de uma explicação. Em outras palavras, através de inscrições digitais e da interação com elas, um cientista, numa reunião de trabalho, tenta convencer os outros de quais são as relações significativas entre os diferentes processos de interesse.

O exemplo abaixo mostra Luke explicando os resultados de um experimento in vivo, no qual um modelo animal (cachorro) foi submetido ao tratamento com o laser. Os resultados foram gravados para processamento futuro nos supercomputadores, e foram exaustivamente debatidos nas reuniões. Luke foi o único do grupo a estar fisicamente presente no hospital durante o experimento, mas todo o procedimento foi observado pela equipe (incluindo o antropólogo) através de imagens e dados numéricos transmitidos via computador. A necessidade de "explicar" o que está acontecendo nas imagens é aqui mais crucial do que em outras instâncias, por causa da ausência física dos outros cientistas no decorrer do experimento. Além disso, Luke foi o cientista responsável por desenvolver as visualizações que expressam aqui os resultados em questão, tendo, portanto, uma intimidade com as imagens e seus significados que não é compartilhada com os outros que, todavia, precisam inteirar-se dos resultados a fim de que se dê continuidade ao projeto como um todo.

O modelo computacional é composto de códigos escritos por vários membros da equipe, que foram depois aperfeiçoados por Luke. A tese de doutorado de Luke, no entanto, trata exclusivamente do manejo do código, tornando-o assim responsável pelo mesmo numa proporção maior do que a de outros cientistas. Alguns até o chamavam de "guardião do código" 
em entrevistas, tarefa que seria herdada por outra pessoa quando ele se formasse. Luke tinha, portanto, a responsabilidade do que foi chamado por todos de "experimento canino" (dog experiment), fazendo viagens ao hospital de pesquisa regularmente e cuidando da logística dos experimentos com animais vivos em termos de tempo de transferência de dados (crucial num experimento que seria acompanhado online em tempo real pelos outros cientistas), corrigindo o sistema de transferência de informações e o código que produziria as previsões matemáticas, a fim de ter certeza de que a equipe poderia ver exatamente o que estava esperando.

Luke negociou com os pesquisadores do hospital a disponibilidade de um cachorro vivo para o experimento e ficou, antes de os experimentos de fato acontecerem, responsável por dar à equipe atualizações a respeito do que poderia ser previsto. Ele também foi responsável por coordenar vários "testes secos", ou testes nos quais o laser era usado em objetos inanimados, para fins de testagem do sistema sem envolver modelos animais vivos. Nestes testes eram utilizados recipientes com gel, nos quais estava embutida uma estrutura oval no formato aproximado de uma próstata. Outros testes usaram uma próstata canina embutida no gel. A opção por modelos caninos visava garantir alguma medida de comparação com os testes nos animais vivos, os chamados testes "para valer".

O trecho a seguir mostra o momento que foi percebido pela equipe como o ápice dos dois anos anteriores da pesquisa, o experimento mais importante até então. Um modelo animal vivo foi submetido ao procedimento completo, incluindo a ressonância magnética pré-operatória, a produção de um modelo digital em 3D da sua próstata, o aquecimento da sua próstata pelo laser e a visualização de todo o procedimento em tempo real, enquanto os dados eram transmitidos para os cientistas. Os dados foram enviados para os supercomputadores, que geraram uma previsão do dano a ser causado e mandaram esses dados de volta ao hospital, completando assim o circuito de transmissão de dados pretendido pelo projeto. Todos os dados e as imagens estavam também disponíveis nos computadores dos cientistas da equipe. Após ser realizado com sucesso, o experimento foi objeto de discussão do grupo: 
Exemplo 7: localizando a próstata na imagem

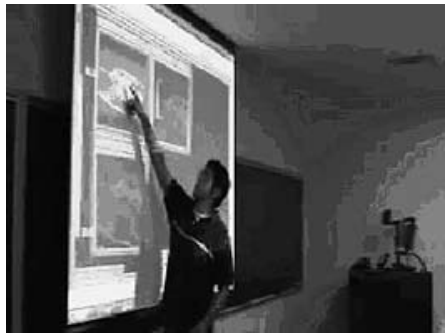

Luke: O que estamos vendo aqui, aqui está a próstata canina ((Luke aponta para a tela, mostrando a janela na qual está a imagem de ressonância magnética da próstata)). ${ }^{13}$

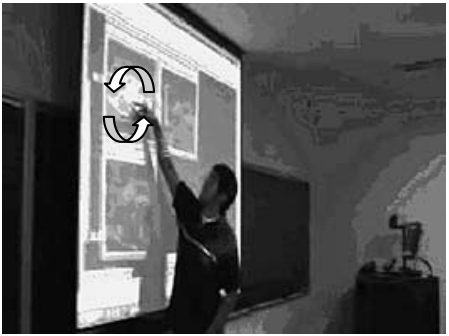

((Em seguida, ele faz um movimento com o dedo, sugerindo uma área circular ao redor do local que havia apontado previamente)). ${ }^{14}$

O exemplo acima mostra como todos os elementos em discussão até aqui funcionam em conjunto nas práticas dos cientistas. Vemos Luke mostrando imagens de ressonância magnética do cachorro e localizando nas imagens o local onde está a próstata, estabelecendo assim o contexto para toda a sua explicação. Nesse momento, somos compelidos a "ver" uma próstata "real" através de uma série de imagens mecanicamente produzidas, incluindo ressonâncias e imagens em 3D produzidas posteriormente por Luke.

No exemplo abaixo, Luke explica como a sonda de fibra ótica (que transmite o laser ao tecido) foi inserida no interior da próstata canina gesticulando na direção da imagem exibida na tela:

\section{Exemplo 8: usando gestos para mostrar direção e orientação da sonda}

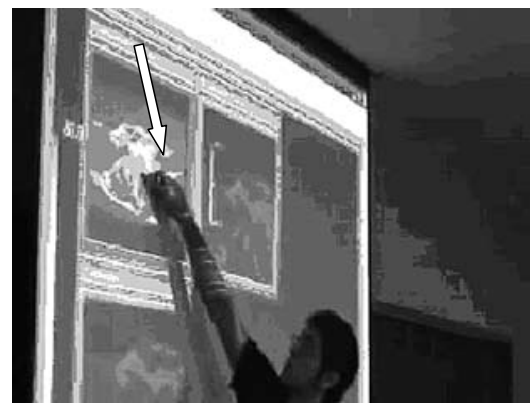


Luke: A fibra intersticial foi inserida nessa direção ((enquanto aponta para a imagem, ele move seus dedos para baixo, sugerindo tanto o ponto de entrada da fibra ótica, quanto o caminho que percorreu até chegar ao ponto onde o aquecimento ocorreria no interior da próstata canina)). ${ }^{15}$

Pode se ver, nos exemplo 7 e 8 acima, quanta informação é transmitida numa duração de aproximadamente 5 segundos, através da combinação de palavras, gestos e imagens. Entende-se imediatamente que o corte transversal mostrado pela imagem de ressonância mostra uma próstata canina; estabelece-se uma área de interesse ao redor de onde a próstata deve estar e entende-se que a fibra ótica foi inserida no tecido num lugar particular, com uma orientação particular, a fim de possibilitar o aquecimento que daria corpo à experiência como um todo.

A imagem apresentada por Luke nestes exemplos tornou-se bastante familiar à equipe de cientistas, pois é muito similar às imagens que ele apresentava regularmente ao grupo na forma de relatórios de progresso, que foi o tipo de apresentação mais comum no período da etnografia. Podemos presumir com certa segurança que os participantes estão a par do fato de que as quatro janelas da tela se referem a diferentes modos de visualização (ressonância magnética, modelo 3D, gráficos de dados experimentais e dados da previsão do computador), sendo também razoável presumir que o grupo entende que os dados que então são apresentados dizem respeito ao experimento com o cachorro.

Ainda que os problemas debatidos nessa reunião tenham sido muito similares àqueles debatidos quando dos experimentos com recipientes de gel, e que os detalhes das imagens não sejam tão claros ao público quanto o são para Luke, desta vez a questão de visualizar com nitidez a próstata e estabelecer a área de aquecimento do laser é de relevância central para a comunicação ao grupo dos resultados da experiência. Os objetos de interesse estão razoavelmente pouco nítidos para a maior parte da equipe, pois nenhum deles possui larga experiência com a análise de imagens de ressonância magnética de cachorros ou de seres humanos.

\section{Conclusão}

Este trabalho analisou gesto e interatividade como relativamente separados um do outro, a fim de enfatizar a especificidade de cada instância de transmissão não-visual de significado a partir de (e em conjunto com) 
visualizações científicas. Tais elementos, no entanto, não ocorrem separadamente na realidade, ou no curso da atividade científica analisada aqui. Ainda que seja analiticamente produtivo focar cada um de forma separada, isto só é verdade quando ajuda a melhor interpretar, de forma conjugada, a rica complexidade dos processos de produção de conhecimento tal qual acontecem na prática.

O objetivo central do texto foi discutir as formas pelas quais o sentido é construído na prática científica, mais especificamente analisando as interações de membros de um grupo de cientistas através da observação participante de suas reuniões semanais. Em tais interações, eles compartilham dados coletados, explicam metodologias e premissas, propõem interpretações a respeito das realidades observadas, construindo, desta forma, um conjunto de significados e objetivos compartilhado por eles. No caso aqui discutido, esses objetivos referem-se à construção de um modelo computacional de transferência de calor em tecidos da próstata capaz de prever dano causado por abrasões a laser. Esse modelo é pensado como parte de um novo tipo de intervenção cirúrgica destinado a melhorar o tratamento do câncer de próstata.

A importância de analisar as formas pelas quais sentidos são produzidos e transmitidos em tais contextos vai além dos conteúdos em debate e das formas específicas pelas quais eles são transmitidos (através de visualizações, gestos e interações). Refere-se também à legitimidade obtida por tais saberes, uma vez que são considerados suficientemente "objetivos" e ao fato de que serão a base para novas formas de intervenção cirúrgica. Esses saberes e significados a respeito do corpo, da próstata e das maneiras com as quais o calor afeta os tecidos farão parte de novas formas de intervenção em nossos corpos materiais, ajudando a determinar o que a ciência considera como saberes adequados (em oposição a interpretações subjetivas) e visualizações objetivas de fenômenos biológicos.

Para contribuir com esta discussão, a metodologia empregada aqui tenta ir além do estritamente visual no seu debate sobre o papel e o uso de visualizações científicas. Como outros autores vêm discutindo em diferentes disciplinas afins, por exemplo, a geografia (Kwan 2002, 2007), existem limites para um paradigma representacional na análise de práticas de visualização e de nossas relações com elas. O argumento aqui proposto é o de que significados e verdades a respeito de processos biológicos, da forma como são tornados aparentes por visualizações científicas, são produzidos por complexos processos interativos envolvendo gestos, manipulação de artefatos digitais, imagens e explanações verbais.

Argumentou-se também que é necessária uma atenção para esses elementos quando da análise das práticas de construção de sentidos comparti- 
lhados na ciência, pois as análises das formas de construção e transmissão de sentido nas práticas científicas aponta para a importância de se levar em conta a opacidade das visualizações científicas, na medida em que não são representações transparentes do real. O sentido dessas visualizações e inscrições é construído através de complexas interações e não dado a priori por qualidades intrínsecas. Busca-se, assim, auxiliar na pesquisa continuada das formas pelas quais tais sentidos são produzidos e transmitidos em práticas científicas e além delas, pois tais afirmações de verdade são instrumentais na definição de como conhecemos nossos corpos e de como desenvolvemos formas de manipulá-los.

Marko Monteiro é pós-doutorando no Departamento de Política Científica e Tecnológica da UNICAMP. E-mail: <markosy@uol.com.br>

\section{Notas}

${ }^{1}$ Exemplos desse tipo de relação prática entre modelos e intervenção na realidade incluem desde previsões climáticas, que afetam a elaboração de políticas ambientais, até previsões de susceptibilidade a doenças a partir de análises bioinformáticas, que influenciam o tratamento de doenças e a elaboração de remédios.

${ }^{2}$ Todos os nomes foram substituídos por pseudônimos a fim de proteger a identidade dos cientistas.

${ }^{3}$ Lista de convenções de transcrição:

[ início de sobreposição de falas;

] fim de sobreposição de falas;

(0.0) tempo transcorrido, em décimos de segundos;

, a vírgula indica uma quebra entre falas muito curta para ser medida, provavelmente uma pausa muito curta;

(.) uma quebra de aproximadamente um décimo de segundo;

. queda de tom indicando fim, não necessariamente fim de frase;

(?) ininteligível; 
(palavra) audição duvidosa;

(( ))descrições da pessoa que transcreveu, ao invés de (ou acrescentando à) fala transcrita.

4 "Lynn: The whole point of our model right is to model the uh, changing, temperature throughout the prostate, this is the heat transfer, model and hum (.) we understand that, temperature comes as a change in energy. (.) ((turns gaze from audience to the screen behind her)) hum so, essentially we're looking up here ((walks towards screen and points)) Q should be (.) ((turns back to audience)) a change in the temperature which will some how come from a change in energy".

${ }^{5}$ Todas as reuniões aconteceram em inglês, e todas as traduções são do autor.

${ }^{6}$ Clark: So that's the reason behind ((points to screen)) like taking that shift, where uh (.) this is, [actually],

Mark: [What is (?)]

Clark: uh, I mean

Mark:(.) What is (?) a new function $\mathrm{P}$, what is the old function?

Clark:Yeah, it is actually ((looks and points at screen)) the uh [inverse (?)]

Lewis: [Inverse] Fourier transfer.

${ }^{7}$ Clark: So first of all let's, uh, I mean, I want to review, uh, that, what is image registration (.) So in computer vision (.2) when sets of data acquired by, sampling the same scene (.) or, I mean, so, if you, if we acquire image data (.2) of the same scene, at different times (.) or if we acquire, the data about an image, about the same image from different perspectives, then these two things will be in different coordinate systems. (.) And we need, the image registration is a process of, registering both of them onto a same coordinate system. (.) Uh and it is necessary because in order to compare or integrate those data, uh we need to have them on the same coordinate system. For example, in, our case, where we are doing, uh, where we'll be having prostate images, as we know that, a prior prostate image data will be given to us, uh much prior before the operation. And then, during the day of the operation we'll be getting, uh, prostate data again. So (.) I mean to make sense of the uh, of this data, and to do computations on this data, we need to register this new prostate image on the previous prostate images, which, we have already done some pre-computations on. So, that is why we require image registration of the two prostate images.

${ }^{8}$ So the idea is like to rotate ((performs hand gesture as he points at second image))...

${ }^{9}$... this image so that it overlaps ((hands shift to third, registered image))

10 Clark: So these are the A and the B images (.) and (.) so we apply transformations to $\mathrm{A}$

((as he says this he clicks on this window with the mouse, causing it to come to the foreground on the screen. He then moves the mouse to use the tools of the software to check the values of $\mathrm{x}$ )) 
(.13) As we said X was 15.

((he changes a variable on the image, causing it to change color)) (.14) and uh (.) $\mathrm{Y}$ was 47

((moves the mouse to check the values of Y)) (.5)

(...)

Then, uh, this image compare too has actually superimposed both the images. (.) ((he walks away from the console and towards the screen, pointing to bottom image)) This one is the transformed image, and this one ((points to image on top right)) is the B image, and ((he walks back to console)), if we [uh]

Luke: [could] you shift the bottom, window, up a little?

Clark: Ok.

((he clicks and shifts the window up, making more of the image visible on the screen)) (.14)

((during this time he is shifting the image and also moving the pointer around to show the two superimposed images))

$\mathrm{ok},(?)(.8)$

((he keeps shifting the images in the bottom window, moving it from left to right, exposing how one part of the image is superimposed on top of the other))

${ }^{11}$ Luke: Yeah so, I mean (.) all we've set up basically is just a way of comparing the two images to, kind of visually uh, ((while he is talking he is dragging the images up and down in the screen)) confirm that, the code is, doing what it's supposed to be doing. So if you look at hum ((he manipulates the bottom image, and now in place of the vertical line separating both parts of the image, we have a round opening that exposes the separation between the two superimposed images. By manipulating it he makes this round opening smaller or larger, to show how the image on top matches the image under it))...

12 ... at, slice, b right here, and then, hum, the, brighter circle in the middle is the, registered slice A, and hum, ((he opens and closes the circle, exposing the superimposition of the images)) you could see that the code is (.) is kind of visually doing what we would expect.

${ }^{13}$ Luke: What we're looking at, here's the canine prostate. ((Luke points to the screen, to the window where the MRI images are.)).

${ }^{14}$ ((He then makes a movement with his finger suggesting a round shape, or a circle around the area where he had previously pointed to.)).

${ }^{15}$ Luke: The interstitial fiber was inserted in this direction. ((as he is pointing to the image, he moves his finger downwards, suggesting both a point of entry where the fiber was inserted and a path it followed as it reached the point where the heating would occur inside the dog's body)). 


\section{Referências bibliográficas}

ALAČ, Morana \& HUTCHINS, Edwin 2004. "I see what you are saying: action as cognition in MRI brain mapping practice". Journal of Cognition and Culture, 4(3):629-661.

BECVAR, L. Amaya, HOLLAN, James et alli. 2005. "Hands as molecules: representational gestures used for developing theory in a scientific laboratory". Semiotica, 156(4):89-112.

BEMENT JR., Arden. 2007. Cyberinfrastructure visions for 21st century discovery. Arlington: National Science Foundation.

CHADAREVIAN, Soraya \& HOPWOOD, Nick. (orgs.). 2004. Models: the third dimension of science. Stanford: Stanford University Press.

CORRIE, Brian \& STOREY, Margaret-Anne. 2007. "Toward understanding the importance of gesture in distributed scientific collaboration". Knowledge and Information Systems, 13:143-171.

CSORDAS, Thomas. 1990. "Embodiment as a paradigm for anthropology". Ethos, 18:5-47.

DASTON, Lorraine \& GALISON, Peter. 1990. "The image of objectivity". Representations, 40:81-128.

FRANCOEUR, Eric \& SEGAL, Jerome. 2004. "From model kits to interactive computer graphics". In: S. d. Chadarevian \& N. Hopwood (orgs.), Models: the third dimension of science. Stanford: Stanford University Press. pp. 402-433.

GOODWIN, Charles. 1994. "Professional vision". American Anthropologist, 96(3):606-633.

HOPWOOD, Nick \& CHADAREVIAN, Soraya. 2004. "Dimensions of modeling". In: . (orgs.), Models: the third dimension of science. Stanford: Stanford University Press. pp. 1-19.
KNORR-CETINA, Karin \& AMANN, Klaus. 1990. "Image dissection in natural scientific inquiry". Science, Technology and Human Values, 15(3):259283.

KRAUT, Robert, FUSSELL, Susan et alli. 2003. "Visual information as a conversational resource in collaborative physical tasks". Human-Computer Interaction, 18:13-49.

KWAN, Mei-Po. 2002. "Feminist visualization: re-envisioning GIS as a method in feminist geographic research". Annals of the Association of American Geographers, 92(4):645-661. . 2007. "Affecting geospatial technologies: toward a feminist politics of emotion". Professional Geographer, 59(1):22-34

LATOUR, Bruno. 1990. "Drawing things together". In: M. Lynch \& S. Woolgar (orgs.), Representation in scientific practice. Cambridge: MIT Press. pp. 19-68.

. 1995. "The 'pedofil' of Boa Vista: a photo-philosophical montage". Common Knowledge, 4(1):144-187. . \& WOOLGAR, Steve. 1986. Laboratory life: the construction of scientific facts. Princeton: Princeton University Press.

LYNCH, Michael. 1990. "The externalized retina: selection and mathematization in the visual documentation of objects in the life sciences". In: M. Lynch \& S. Woolgar (orgs.), Representation in scientific practice. Cambridge: MIT Press. pp. 153-186. . 2006. "The production of scientific images: vision and re-Vvision in the history, philosophy and sociology of science". In: L. Pauwels (org.), Visual cultures of science: rethinking repre- 
sentational practices in knowledge building and science communication. Hanover: Dartmouth College Press. pp. 26-41.

LYNCH, Michael \& WOOLGAR, Steve. 1990. "Introduction: sociological orientations to representational practice in science". In: M. Lynch \& $\mathrm{S}$. Woolgar (orgs.), Representation in scientific practice. Cambridge: MIT Press. pp. 1-19.

. \& WOOLGAR, Steve. (orgs.). 1990. Representation in scientific practice. Cambridge: MIT Press.

MYERS, Natasha. 2007. Modeling proteins, making scientists: an ethnography of pedagogy and visual cultures in contemporary structural biology. Science, technology and society. PhD Dissertation, Massachusetts, Institute of Technology.

- 2008. "Molecular embodiments and the body-work of modeling in protein crystallography". Social Studies of Science, 38(2):163-199.

OCHS, Elinor, JACOBY, Sally et alli. 1994. "Interpretive journeys: how physicists talk and travel through graphic space". Configurations, 2(1):151-171.

1996. "'When I come down I'm in the domain state': grammar and graphic representation in the interpretive activity of physics". In: E. Ochs, E. Schlegloff \& S. Thompson (orgs.), Interaction and grammar. Cambridge: Cambridge University Press. pp. 328-69.

PASVEER, Bernike. 2006. "Representing or mediating: a history and philosophy of x-ray images in medicine". In: L. Pauwels (org.), Visual cultures of science: rethinking representational practices in knowledge building and science communication. Hanover: Dartmouth College Press. pp. 41-63.
PAUWELS, Luc. (org). 2006. Visual cultures of science: rethinking representational practices in knowledge building and science communication. Hanover: Dartmouth College Press. SCHNALKE, Thomas. 2004. "Casting skin: meanings for doctors, artists, and patients". In: S. d. Chadarevian \& N. Hopwood (orgs.), Models: the third dimension of science. Stanford: Stanford University Press. pp. 207-242. 


\section{Resumo}

O objetivo deste trabalho é discutir como objetos virtuais participam interativamente da produção do conhecimento na prática científica. $\mathrm{O}$ artigo baseia-se numa observação etnográfica de uma equipe interdisciplinar de cientistas, cujo trabalho envolve modelagem computacional de transferência de calor na próstata humana. A etnografia constatou que, embora visualizações científicas sejam pensadas como uma forma de "simplificar" a apreensão de dados, há um intenso trabalho interpretativo necessário para alcançar sentidos compartilhados a respeito das imagens. Tais sentidos são construídos a partir de comunicação oral e de interações incorporadas com objetos virtuais no decorrer das interações entre os cientistas. Uma melhor compreensão dessas práticas interpretativas é importante na medida em que o uso de visualizações digitais em 3D e de modelos computacionais ganha importância na ciência contemporânea. Tais técnicas são crescentemente utilizadas não somente para descrever verdades sobre a natureza, mas como ferramentas poderosas de intervenção no mundo.

Palavras-chave Modelagem computacional, Gesto, Interação, Etnografia da ciência, Representações visuais, Tecnologias digitais

\section{Abstract}

This text discusses how virtual objects participate interactively in the production of knowledge in scientific practice. The article is based on the ethnographic observation of an interdisciplinary team of scientists whose work involves computer modelling of heat transfer in the human prostate. The ethnography found that although scientific imaging may be considered a form of 'simplifying' the apprehension of data, an intense interpretative process is required to achieve shared meanings concerning the images. These meanings are constructed through oral communication and embodied interactions with virtual objects during the interactions between scientists. A better understanding of these interpretative practices is needed given the growing importance of the use of 3D digital imaging and computational models in contemporary science. These techniques are increasingly used not only to describe truths about nature, but also as powerful tools for intervening in the world.

Key words Computational modelling, Gestures, Interaction, Ethnography of science, Visual representations, Digital technologies 